\title{
New Derivatization Reagent for Detection of free Thiol-groups in Metabolites and Proteins in Matrix-Assisted Laser Desorption/lonization Mass Spectrometry Imaging
}

Annabelle Fülöp, ${ }^{1 *}$ Tobias Bausbacher, ${ }^{1}$ Stefano Rizzo, ${ }^{2}$ Qiuqin Zhou, ${ }^{1}$ Hartmut Gillandt, ${ }^{2}$ Carsten Hopf, ${ }^{1}$ and Miriam Rittner ${ }^{2}$

${ }^{1}$ Center for Mass Spectrometry and Optical Spectroscopy (CeMOS), Mannheim University of Applied Sciences, Paul-Wittsack-Str. 10, 68163 Mannheim, Germany

${ }^{2}$ Sirius Fine Chemicals SiChem GmbH, Fahrenheitstr. 1, 28359 Bremen, Germany

\section{SI table of contents}

\begin{tabular}{|l|l|l|}
\hline SI Experimental Section & $\begin{array}{l}\text { Materials. Synthesis and purification of CHC-Mal. } \\
\text { Animal Model and Tissue Samples. Hematoxylin and } \\
\text { Eosin tissue staining. Sample handling and TCEP } \\
\text { reduction. }\end{array}$ & S-2 \\
\hline SI Figure 1 (Fig. S1) & Chemical structure of CHC-Mal. & S-5 \\
\hline SI Figure 2 (Fig. S2) & H\&E stained porcine pancreas section. & S-5 \\
\hline SI Figure 3 (Fig. S3) & Single pixel spectra and derivatization rates from Fig. 1. & S-6 \\
\hline SI Figure 4 (Fig. S4) & $\begin{array}{l}\text { Positive MALDI MSI of GSH, GSSG and derivatized } \\
\text { GSH on pig liver tissue and pipetted GSH solution on } \\
\text { ITO glass slide }\end{array}$ & S-6 \\
\hline SI Figure 5 (Fig. S5) & MS/MS spectra of m/z 745.19 ([GSH-CHC+K] $\left.]^{+}\right)$ & S-7 \\
\hline SI Figure 6 (Fig. S6) & Comparison of H\&E stained tumor samples. & S-7 \\
\hline SI Figure 7 (Fig. S7) & $\begin{array}{l}\text { Application of CHC-Mal do not cause delocalization of } \\
\text { endogenous lipids or metabolites after derivatization of } \\
\text { tissue sections. }\end{array}$ & S-8 \\
\hline
\end{tabular}




\section{SI Experimental Section}

\section{Materials.}

Chemicals for the synthesis of CHC-Mal were purchased from Sigma Aldrich and used without further purification. Solvents were generally obtained from VWR (Bruchsal, Germany) and Sigma Aldrich in the highest available purity. ${ }^{1} \mathrm{H}$ - and ${ }^{13} \mathrm{C}$-NMR spectra were recorded on Bruker Avance Neo $(600.22$ $\mathrm{MHz}(1 \mathrm{H})$ and $150.94 \mathrm{MHz}(13 \mathrm{C}))$ spectrometer. Chemical shifts are expressed in parts per million $(\mathrm{ppm})$ and the spectra are calibrated to residual solvent signals of $\mathrm{CDCl}_{3}(7.26 \mathrm{ppm}(1 \mathrm{H})$ and $77.0 \mathrm{ppm}$ (13C)). Low Resolution Mass Spectra (LR-MS) were recorded on a Bruker amaZon SL ESI spectrometer. The MALDI matrix DHB (2,5-Dihydroxybenzoic acid) and indium tin oxide (ITO)coated conductive glass slides were obtained from Bruker Daltonik GmbH (Bremen, Germany). Tris(2carboxyethyl)phosphine (TCEP) was obtained from Alfa Aesar (Karlsruhe, Germany). Oxygen absorber ATCO FT100 was obtained from Long Life for Art (Eichstetten, Germany).

\section{Synthesis and purification of CHC-Mal.}

$\alpha$-Cyano-4-hydroxycinnamic acid $(0.25 \mathrm{~g} ; 1.32 \mathrm{mmol} ; 1 \mathrm{eq})$ was dissolved in $10 \mathrm{ml}$ of dry $N, N$ Dimethylformamid (DMF), followed by the addition of $N, N, N^{\prime}, N^{\prime}$-Tetramethyl-O-(Nsuccinimidyl)uronium tetrafluoroborate $(0.39 \mathrm{~g} ; 1.32 \mathrm{mmol}, 1 \mathrm{eq})$ and diisopropylethylamine $(0.46 \mathrm{ml}$; $2.64 \mathrm{mmol} ; 2 \mathrm{eq}$ ). The solution was allowed to stir under nitrogen at room temperature for $1 \mathrm{~h}$, after which 1-(2-(2-(2-aminoethoxy)ethoxy)ethyl)-1H-pyrrole-2,5-dione $(0.30 \mathrm{~g} ; 1.32 \mathrm{mmol} ; 1 \mathrm{eq})$ was added. The mixture was stirred for further $16 \mathrm{~h}$, then DMF was removed under vacuum and the residue was purified via reverse-phase chromatography (RPC) on a YMC-Triart C18-S column $(4.6$ x $250 \mathrm{~mm})$ with ultraviolet (UV) detection at $350 \mathrm{~nm}$. Elution was performed isocratically with $50 \%$ methanol/50\% $\mathrm{H}_{2} \mathrm{O}$ with $0.1 \% \mathrm{CF}_{3} \mathrm{COOH}$ as solvent. The product-containing fractions were concentrated under reduced pressure affording (E)-2-cyano- $N$-(2-(2,5-dioxo-2,5-dihydro-1H-pyrrol-1yl)ethyl)-3-(4-hydroxyphenyl)acryl-amide (CHC-Mal) as a beige solid (0.42 $\mathrm{g} ; 1.05 \mathrm{mmol} ; 79 \%) .{ }^{1} \mathrm{H}$ NMR (600 MHz, Chloroform-d) $\delta 8.16(\mathrm{~s}, 1 \mathrm{H}), 7.90-7.80(\mathrm{~m}, 2 \mathrm{H}), 6.97-6.93(\mathrm{~m}, 2 \mathrm{H}), 6.92(\mathrm{t}, \mathrm{J}=$ $5.4 \mathrm{~Hz}, 1 \mathrm{H}), 6.71(\mathrm{~s}, 2 \mathrm{H}), 3.77(\mathrm{t}, \mathrm{J}=5.6 \mathrm{~Hz}, 2 \mathrm{H}), 3.69(\mathrm{t}, \mathrm{J}=5.6 \mathrm{~Hz}, 2 \mathrm{H}), 3.68-3.53(\mathrm{~m}, 8 \mathrm{H}) .{ }^{13} \mathrm{C}$ NMR (151 MHz, Chloroform-d) $\delta$ 170.82, 161.75, 161.11, 152.67, 134.19, 133.50, 124.02, 117.40, 116.46, 99.63, 70.37, 70.01, 69.32, 68.06, 40.37, 37.17. LR-MS (ESI) calc. for $\mathrm{C}_{20} \mathrm{H}_{21} \mathrm{~N}_{3} \mathrm{NaO}_{6}$ $[\mathrm{M}+\mathrm{Na}]^{+}:$422.14, found 422.13 . 


\section{Animal model and tissue samples.}

Pig liver and pancreas tissues were obtained from a slaughterhouse and directly stored at $-80^{\circ} \mathrm{C}$. Animal inoculated and organ removal was conducted by a contract research organization, Heidelberg Pharma GmbH (Ladenburg, Germany). The Heidelberg Pharma GmbH animal test facility was registered by the regional licensing body ("Regierungspräsidium Karlsruhe") and the specific experiment protocol was approved by the ethics committee attached to Regierungspräsidium Karlsruhe. The institutional ethics committee ("Tierschutzausschuss") of Heidelberg Pharma GmbH reviewed and enforced the general compliance with animal welfare of the facility. In brief, female CB-17 SCID mouse (7 weeks) were purchased from Charles River Laboratories (Arbresle, France) and inoculated subcutaneously with NCl-H929 tumor cells into the flank. Mouse was sacrificed after 15 days, tumor sample was snap frozen and stored at $-80^{\circ} \mathrm{C}$.

Frozen tissue was sliced into $12 \mu \mathrm{m}$ sections using a Leica CM1950 cryostat (Leica Biosystems, Nussloch, Germany) at a chamber temperature of $-15^{\circ} \mathrm{C}$. Cryosections were mounted onto ITO glass slides and dried for $30 \mathrm{~min}$ in vacuum. To compare the effects of sample preparation and MALDI MSI measurement, adjacent sections $(12 \mu \mathrm{m})$ were collected and mounted onto HistoBond $\AA+\mathrm{M}$ adhesive microscope slides (Paul Marienfeld GmbH \& Co. KG, Lauda-Königshofen, Germany) and stored at $-80^{\circ} \mathrm{C}$ until tissue staining.

\section{Hematoxylin and Eosin (H\&E) tissue staining.}

After MALDI imaging analysis, used ITO glass slides were washed with pure acetone to remove the matrix and CHC-Mal before histological staining. Tissue sections onto ITO glass slides and adjacent sections mounted onto HistoBond $\mathbb{R}+\mathrm{M}$ adhesive microscope slides were stained together. For Hematoxylin and Eosin staining, the sections were immersed into Mayer's hematoxylin solution (2 $\mathrm{min})$, immersed in tab water ( $3 \mathrm{~min}$ ) and dipped in distilled water afterwards. To enhance the nuclei staining, slides were immersed into $0.3 \%$ acid alcohol ( $0.3 \%$ in $70 \%$ EtOH solution, $30 \mathrm{sec})$, dipped in distilled water, immersed into bluing solution $\left(10 \mathrm{~g} \mathrm{NaHCO}_{3}+100 \mathrm{~g} \mathrm{MgSO}_{4}\right.$ in $\left.5 \mathrm{~L} \mathrm{H}_{2} \mathrm{O}\right)$ for 2 min and rinsed with distilled water. Then, the sections were immersed in Eosin solution for 2 min and rinsed with distilled water. The slices were each dehydrated for $2 \mathrm{~min}$ in various alcohol solutions $(80 \%, 96 \%$, $100 \%$ ) and cleared with xylol solution. Slides were covered with mounting medium (Eukitt) and coverslips. Pictures of H\&E stained histological sections were obtained with an Aperio CS2 Scanner (Leica Biosystems, Wetzlar, Germany) at 20x magnification of the objective. 


\section{Sample handling and TCEP reduction.}

During sample preparation for derivatization of endogenous thiol-containing metabolites the exposure of tissue sections to atmospheric oxygen was minimized. In detail, the tissue sections were stored in containers equipped with oxygen absorbers (ATCO FT100). Chemical compounds were applied to tissue slices by a SunCollect MALDI Spotter (SunChrom, Friedrichsdorf, Germany) with a pressure of 2.5 bar. To avoid excessive thiol oxidation during sample preparation nitrogen was used as spray

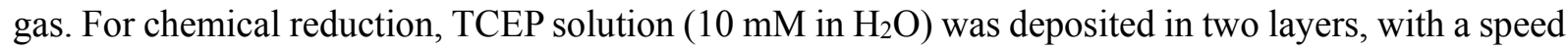
of $300 \mathrm{~mm} / \mathrm{min}$, flow rate of $15 \mu 1 / \mathrm{min}$ and a line distance of $1 \mathrm{~mm}$. The distance between tissue and spray head was $25.3 \mathrm{~mm}$. After deposition of TCEP, glass slides were incubated for $30 \mathrm{~min}$ in a methanol chamber and then dried for $30 \mathrm{~min}$ in vacuum. The complete preparation time for a reduction step using TECP was therefore approximately $75 \mathrm{~min}$ and resulted in a TCEP amount of $10 \mu \mathrm{mol} / \mathrm{mm}^{2}$. 


\section{SI Supplementary Figures}<smiles>N#C/C(=C\c1ccc(O)cc1)C(=O)NCCOCCOCCN1C(=O)C=CC1=O</smiles>

Figure S1: Chemical structure of CHC-Mal.

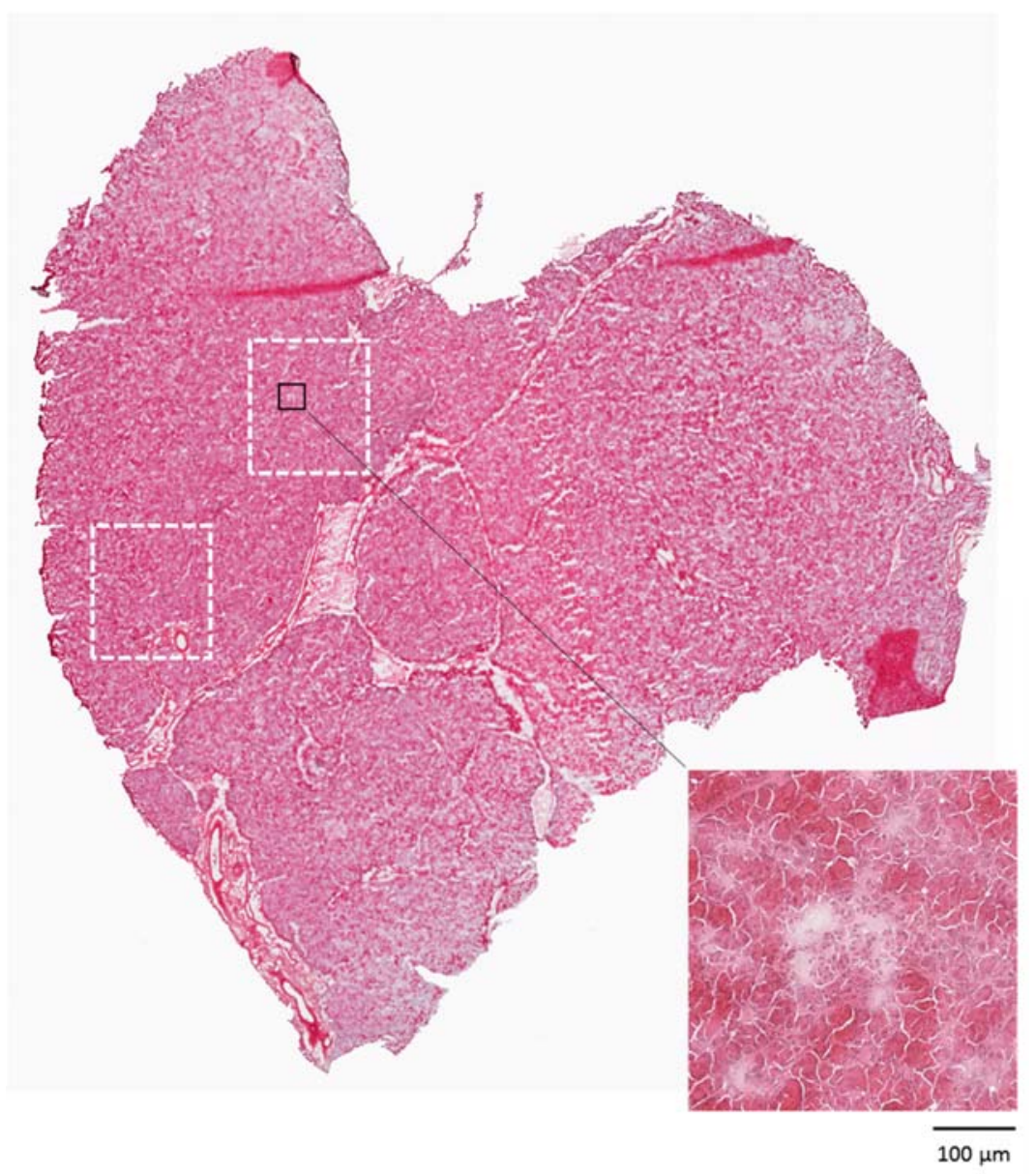

Figure S2: H\&E stained porcine pancreas section.

H\&E staining of the subsequent section of the porcine pancreas shown for the MALDI MSI measurement in Figure 1. White boxes indicate the approximate position of the corresponding measuring regions in Fig. 1 A (the measurements of the underivatized pancreas section and derivatized sections with $0.36 \mu \mathrm{mol} / \mathrm{mm}^{2}$ and $1.04 \mu \mathrm{mol} / \mathrm{mm}^{2}$ CHC-Mal were performed on subsequent tissue sections, so that the measuring region varies slightly from tissue section to tissue section). 

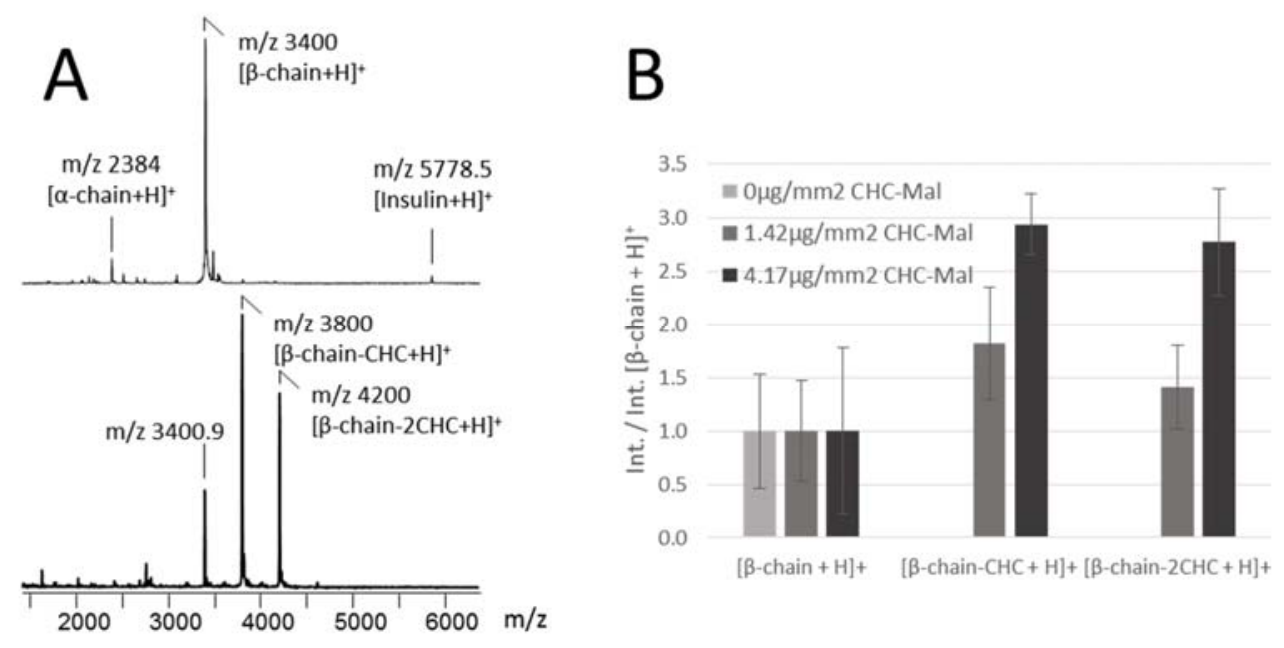

\section{Figure S3: Single pixel spectra and derivatization rates from Fig. 1.}

(A) MALDI MS single spectra of porcine pancreatic sections after reduction with TCEP and in absence (upper spectra) or presence (lower spectra) of a derivatization step with $1.04 \mu \mathrm{mol} / \mathrm{mm}^{2}$ CHC-Mal. (B) Intensity of $[\beta \text {-chain-CHC }+\mathrm{H}]^{+}(\mathrm{m} / \mathrm{z} 3800.3)$ and $[\beta \text {-chain- } 2 \mathrm{CHC}+\mathrm{H}]^{+}(\mathrm{m} / \mathrm{z} 4199.7)$ compared to $[\beta \text {-chain }+\mathrm{H}]^{+}(\mathrm{m} / \mathrm{z} 3400.9)$ in Fig. 1 shows a better derivatization using $1.04 \mu \mathrm{mol} / \mathrm{mm}^{2}$ CHC-Mal. Average values were calculated over all single pixels in Fig. 1.

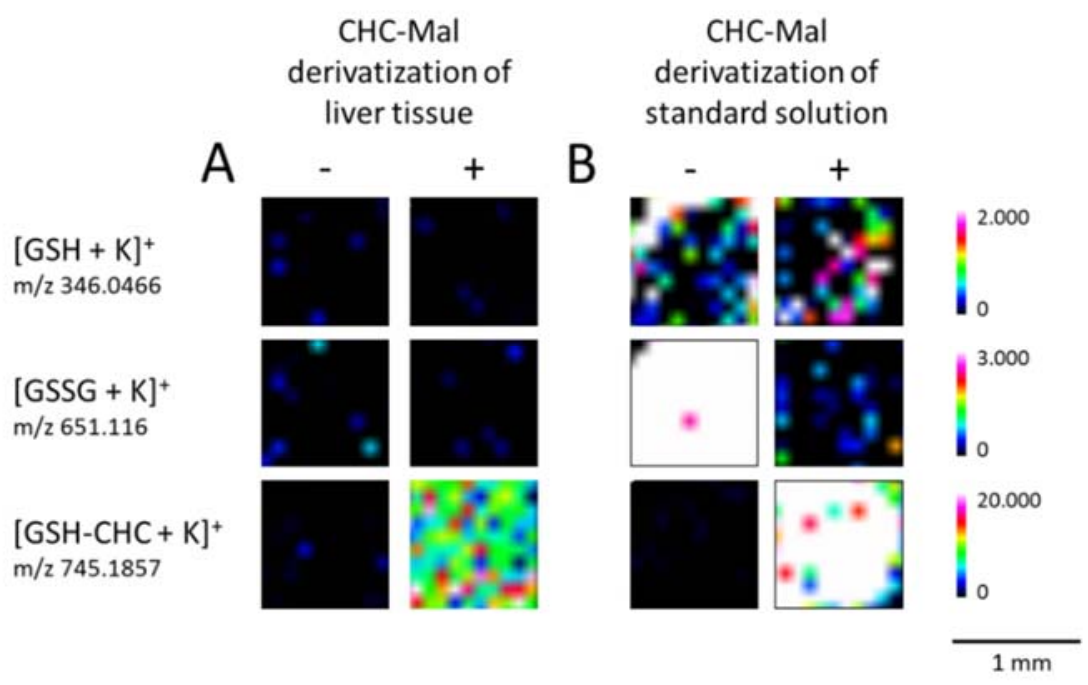

Figure S4: Positive MALDI MSI of GSH, GSSG and derivatized GSH on pig liver tissue and pipetted GSH solution on ITO glass slide.

(A) MALDI MSI of $\mathrm{m} / \mathrm{z}$ signals of reduced (GSH) and oxidized glutathione (GSSG) as well reduced glutathione after derivatization with CHC-Mal (GSH-CHC) in porcine liver slices in absence (left side) or presence (right side) of a derivatization step by CHC-Mal. (B) MALDI MSI of m/z signals of reduced (GSH) and oxidized glutathione (GSSG) as well reduced glutathione after derivatization 
with CHC-Mal (GSH-CHC) after pipetting a solution of GSH (20 nmol) onto the ITO glass slide in absence (left side) or presence (right side) of a derivatization step by CHC-Mal.

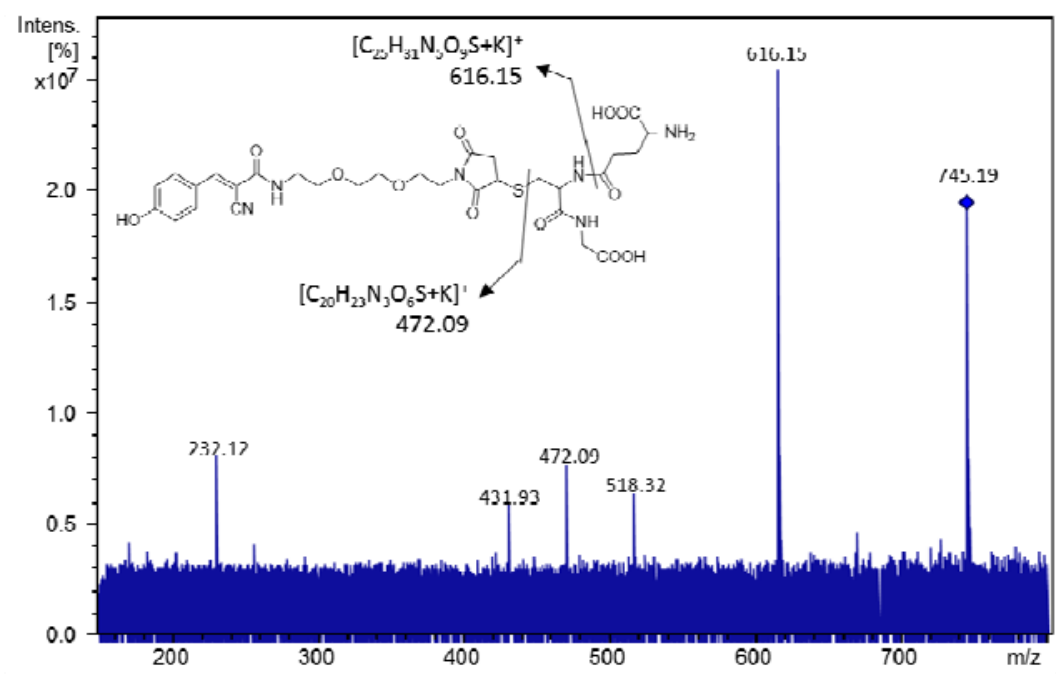

Figure S5: MS/MS spectra of $\mathbf{m} / \mathbf{z} 745.19\left([\mathrm{GSH}-\mathrm{CHC}+\mathrm{K}]^{+}\right)$.

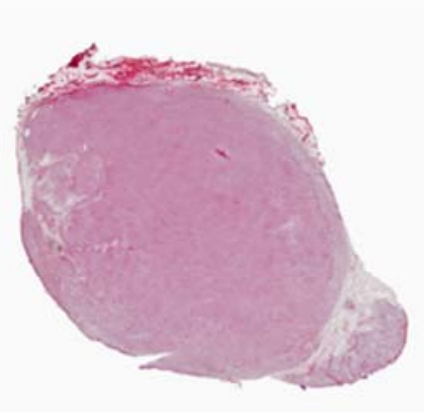

without sample preparation

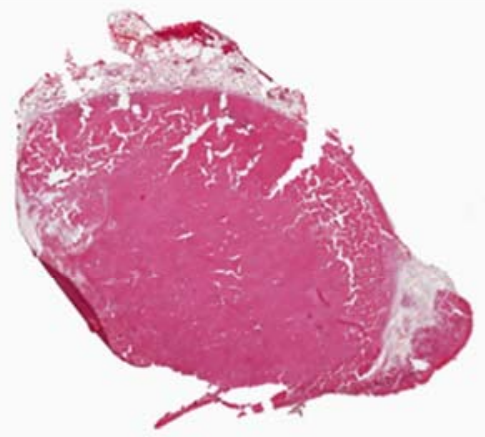

- CHC-Mal

$+\mathrm{DHB}$

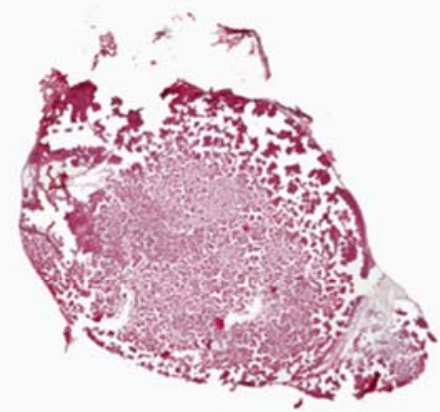

$+\mathrm{CHC}-\mathrm{Mal}$ + DHB

Figure S6: Comparison of H\&E stained tumor samples.

Comparison of H\&E stained tumor samples without sample preparation (left tissue section) and after MALDI MSI measurement of the tumor sample with DHB matrix (middle tissue section) or CHC-Mal and DHB matrix (right tissue section). 

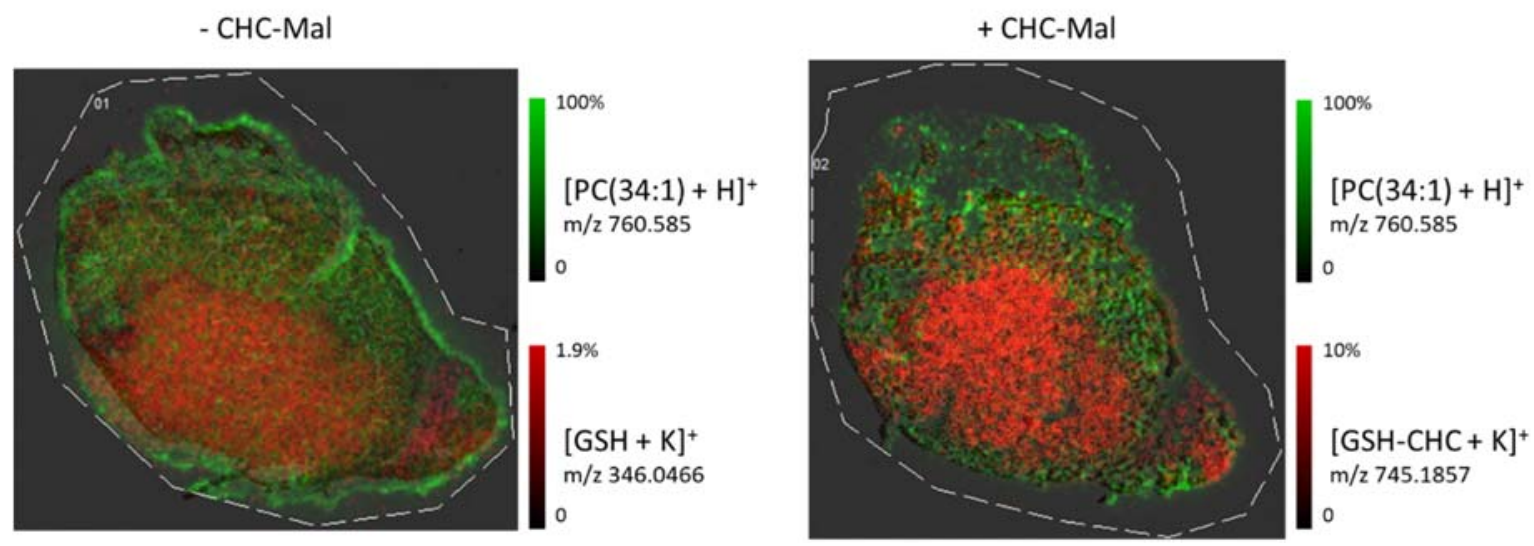

Figure S7: Application of CHC-Mal do not cause delocalization of endogenous lipids or metabolites after derivatization of tissue sections.

Overlay of H\&E stained tumor samples (Fig. S6) with MALDI MSI of m/z signals (Fig. 3) of $[\mathrm{PC}(34: 1)+\mathrm{H}]^{+}$(green color) and $[\mathrm{GSH}+\mathrm{K}]^{+}$or $[\mathrm{GSH}-\mathrm{CHC}+\mathrm{K}]^{+}$(red color) in the absence (left side) or presence (right side) of a derivatization step with CHC-Mal. White marking indicated the MALDI MSI measuring region. 